\title{
Design of Intelligent Parking Lot Based on Radio Frequency Identification
}

\author{
Zuliang Wang ${ }^{1, a^{*}}$, Ting Zhang ${ }^{1, b}$, Ying Yin ${ }^{2, c}$, Xinxin Xie ${ }^{1, d}$, Nana Wan ${ }^{1, e}$ \\ ${ }^{1}$ Department of Information Engineering, Xijing University, Xi'an, 710123, China \\ ${ }^{2}$ School of Electrical Engineering, Xinjiang University, Urumqi 830047, China \\ a1601252678@qq.com, b1046935520@qq.com, c1230256781@qq.com, d1547878322@qq.com, \\ e1763422453@qq.com
}

Keywords: Radio frequency identification; Intelligent parking lot; Anti-collision; Simulation

\begin{abstract}
With the increase of car ownership in our country, the traditional manual management of the parking lot is time-consuming and its efficiency is low, also cannot make full use of parking spaces, resulting in waste of resources. This paper designs a kind of intelligent parking management system based on radio frequency identification technology. Group binding strategy is employed to solve the accounting and parking management problem when multi cars enter the parking space at the same time.
\end{abstract}

\section{Introduction}

With the rapid growth of the national economy, the car ownership increases, the way people go out tend to self-driving, at the same time, perception accuracy, timing and charging need manual participation questions appear in parking lot. Because of the large number of vehicles, there will many vehicles to enter the parking lot at the same time, so automatically statistics vehicle information and timing charges cannot be realized. Therefore, it is of great significance to design a kind of intelligent parking system which can accurately perceive the parking space information, and can solve the problem of multi vehicle access at the same time. The existing intelligent parking management system can accurately perceive parking and vehicle information management when a single vehicle enters into the parking lot, but if many vehicles entering, management and charging process need manual intervention, which is inconvenient. Based on RFID and geomagnetic sensor technology an intelligent parking space sensing and timing management system is designed. The main contribution of the paper is to improve the anti-jamming capability of the geomagnetic sensor recognize the car entering into the parking space, and RFID tag discriminative ability, especially when more than one car into the sensing range at the same time, how to solve the vehicle timing and charging and parking status recognition.

\section{System Design}

The structure of intelligent parking management system is shown in Fig. 1. The system is composed of a parking status acquisition module (make of two parts: a geomagnetic sensor and EndDevice node), a communication module based on Zigbee technology, and a timing and charging module based on RFID. The communication module based on Zigbee technology is made up of three communication nodes, that is, EndDevice node, Router node and Coordinator node. Parking status acquisition module, vehicle ID identification and timing and charging module are all communicate with the Zigbee wireless communication network, and Coordinator node of Zigbee wireless network is connected with the background server. 


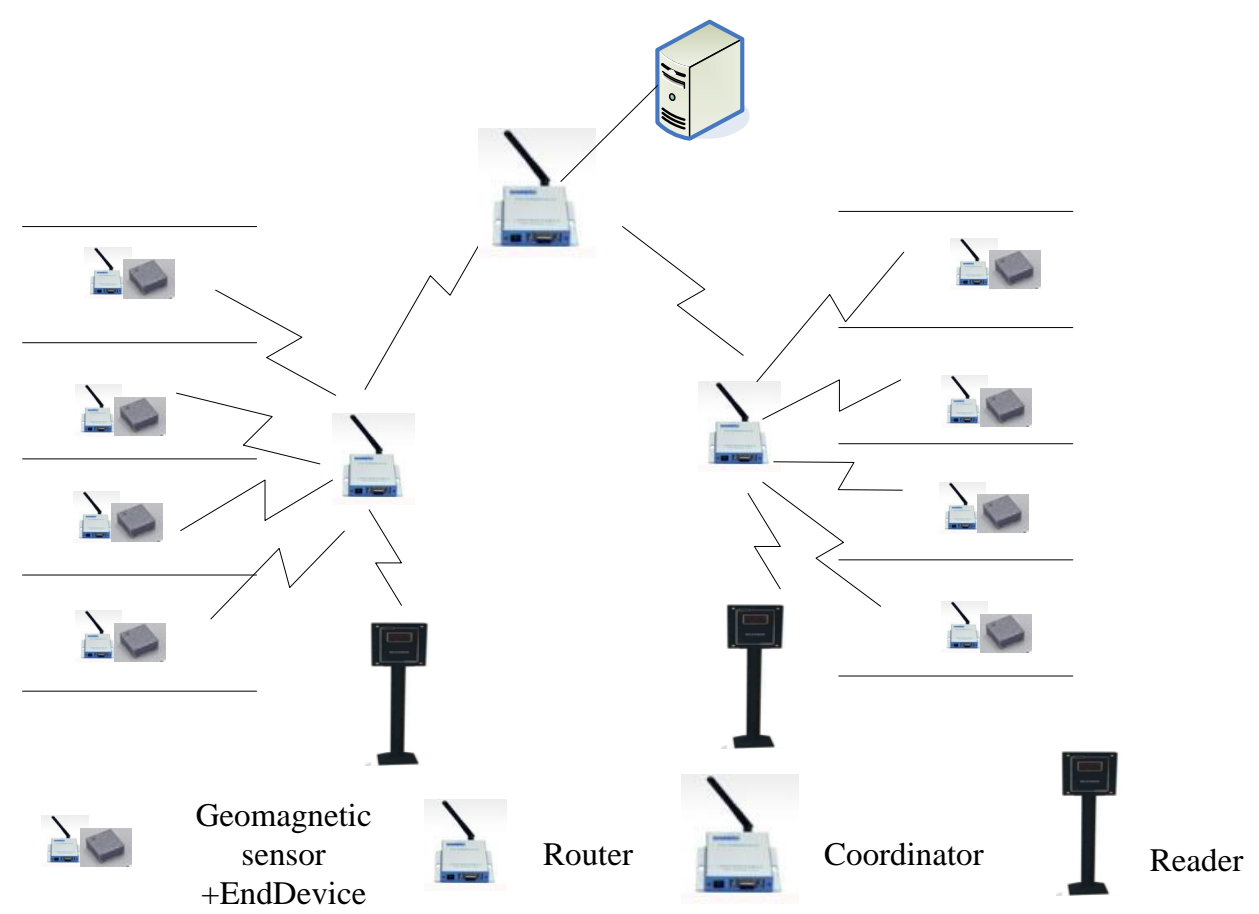

Figure 1. Structure of intelligent parking management system

Parking status acquisition module uses geomagnetic sensors to detect the occupancy of parking space. When it detects the vehicle occupied parking lot, then informing the backend server of parking occupancy through Zigbee's three level nodes, and recognize vehicle ID information through the RFID reader, complete the binding between vehicle parking and ID information, and timing. If vehicle is detected runs out of parking spaces, then reporting the parking space free to the backend server and charging.

\section{Algorithm Steps}

When there are $\mathrm{N}$ cars parked at $\mathrm{N}$ parking spaces at the same time, RFID reader cannot recognize the corresponding relation of the car into a specific parking spaces. In this case, the $\mathrm{N}$ cars and the corresponding occupied $\mathrm{N}$ parking spaces are bound as a group. When one car left, the vehicle ID and parking spaces can be recognized through the geomagnetic sensor and RFID together, complete the timing and charging correctly and recognize the parking space is free, and remove the parking space and vehicle number ID from their binding group. When the vehicle left in this group again when, repeat the above process until only one car and a parking space left, then it can be transformed into one to one binding, cancel the group binding, realize the accurate vehicle information management and charging when multiple vehicles enter at the same time. The system can recognize parking space intelligently for open parking lots, and it has the advantages of precision timing and charging automatically, also can save human resources and so on. The processing steps are as follows.

Step 1: Collect parking information by magnetic sensor, and determine whether the car is leaving or entering. If the car is entering, turn to step 2, otherwise, if the car is leaving turn to step 6.

Step 2: Start RFID to identify the number of cars, if there is only one car to enter the identification zone at the same time, turn to step3, and otherwise turn to step4.

Step 3: The main module binds the car ID and parking space, and turn step 8.

Step 4: Bind the new arrival car group and the newly occupied parking space group, and go to step 8.

Step 5: Send car departure information to the control center. If it is group binding, then turn to

step 7, and otherwise turn to step 6.

Step 6: Remove the car-parking space binding, start RFID charges, update the status of the corresponding parking space as empty, and turn to step 8 . 
Step 7: Start RFID charges, the number of members group minus one until the group members reduced to 1 , and change to one to one binding.

Step 8: End.

Tag Anti Collision Method

In intelligent parking management system designed in this paper, in the same coverage of RFID reader, when there are more than one car into the parking space at the same time, RFID reader needs to identify multiple tags at the same time. At present, passive RFID tag has been widely used because of its low cost, no power supply and so on. However, the computing ability of passive tag is limited, they cannot induct channel, and perceive the collision or communicate with other tag. Once it receives the reader instructions, and it will send a response immediately, so the collision will inevitably happen. A certain method is needed to reduce tag collision and improve the recognition speed and efficiency.

RFID anti collision algorithm has such three kinds that are ALOHA algorithm, the algorithm based on the binary tree and hybrid algorithm. AOHA algorithm is divided into pure ALOHA (PA, Pure, ALOHA), sequence ALOHA (SA, Slot ALOHA), frame timing ALOHA (FSA, Frame Slot ALOHA) and so on. FSA also can be divided into basic frame sequence of ALOHA (BFSA, Basic Frame Slot ALOHA) and dynamic frame timing ALOHA (DFSA, dynamic frame Slot ALOHA). EPC_C1 G2 standard uses DFSA method to prevent tag collision. EPC_C1 G2 standard is widely used in the field of radio frequency identification. In this paper, the performance of EPC_C1 G2 standard anti collision algorithm is evaluated, so as to provide theoretical basis for the design of intelligent parking lot and parameter. The examples of tag anti collision algorithm are shown in Fig. 2. Fig. 3 is the anti collision algorithm model according to G2 EPC_C1 standard, including 5 tags, and the initial frame length is 5 slots.

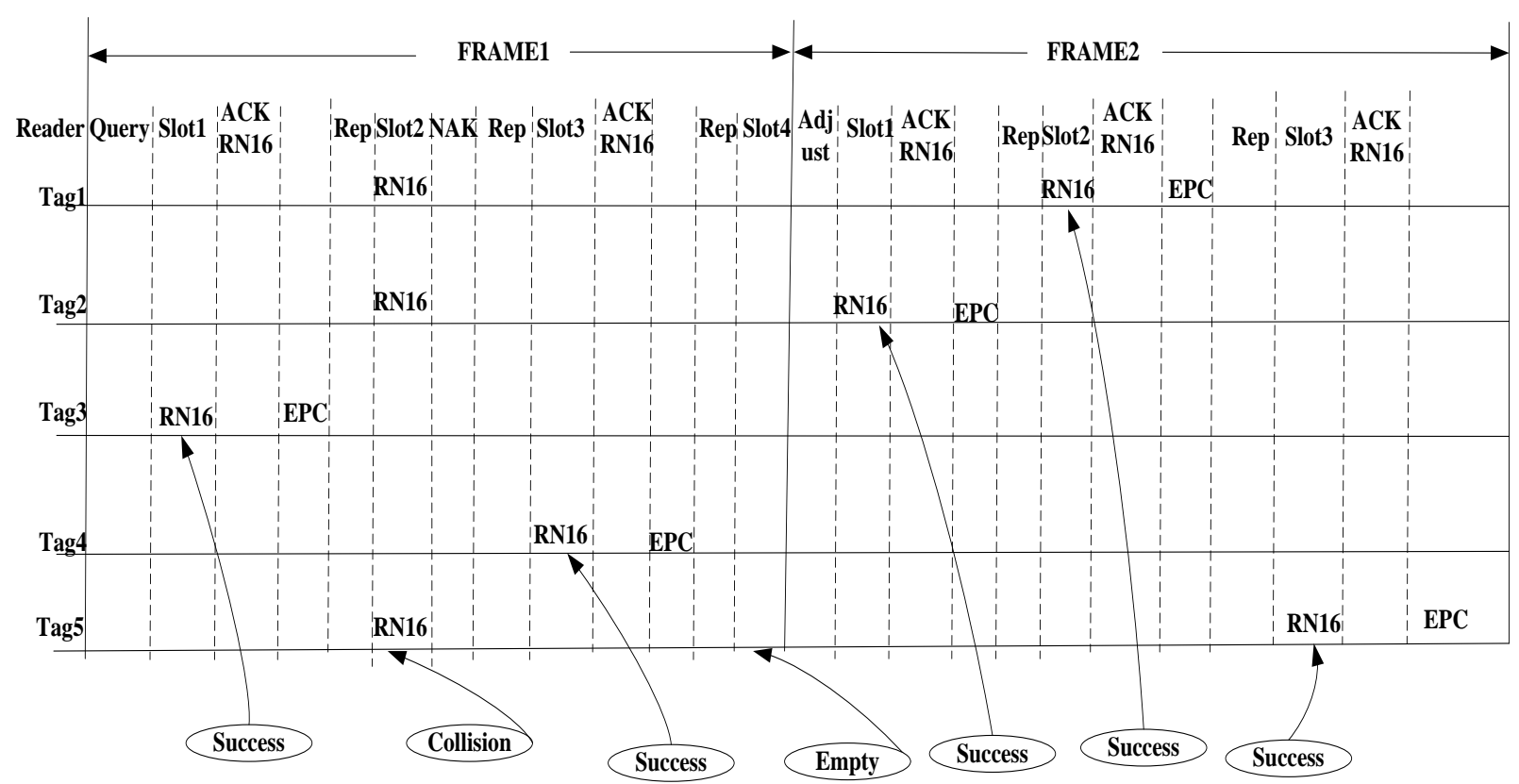

Figure 2. EPC_C1 G2 tag anti collision algorithm example

Once the tags sense the reader, they are ready to enter the ready state, waiting for the reader to order. After a certain period of time, the reader sends a Query command that contains the Q parameter of the frame length, and a random number generator generates a random number in the $0 \sim \mathrm{Q}$ range, and the random number is stored in the counter. If the random number is 0 , the RN16 random number is sent immediately.

The tag information that the reader receives has three cases:

(1) If only one tag sends RN16 in the time slot, for example in Fig. 2, only the tag3 label sends data in the slot1 time slot, so the reader can receive RN16 correctly. After a certain time, the reader send ACK confirming the information with the RN16 label to the tags, the tag2 label receives this 
information, consistent with the RN16 on this label previously sent, a communication link is established, then can read tag data. For example in Fig. 2, tag3 tag sends EPC (electronic products encoding) to the reader. The remaining label did not send RN16, so cannot accept the ACK message. After completing a full tag identification, the reader sends KILL instruction to the tag, and then the tag enters the silent state and is not involved in the subsequent tag identification process. The tags of random number greater than 0 into the ARBITRATE state waiting for the subsequent Rep command or Adjust command. Upon completion of a slot, the reader sends a Rep command to open a new slot. All the counters of survival tags will be reduced by 1 after the Rep command is received, if the tag random number is 0, send the RN16 immediately, or enter the ARBITRATE state.

(2) If the reader does not receive any response from the tag in a slot, the slot is an empty slot, such as the slot 4 slot shown in Fig. 2. At this point, the reader immediately sends a Rep command to open a new time slot.

(3) If there is an extra tag sends RN16 in a slot, then a collision occurred, such as tag tag1, tag2 and teg 5 the three tags in the slot 2 time slot send the RN16 to the reader at the same time shown in Fig. 2. The reader detected time slot collision using Manchester encoding, then sent NAK information to three labels (tag1, tag2 and teg5) in the time slot sending RN16, and points out that the time slot collision occurred, and send Rep command to all surviving label Rep commands to open a new slot. The collision tags received NAK information to set the slot counter as 7FFF, and enter the ARBITRATE state to wait for the Adjust command to participate in the identification of a new frame.

After accomplish the frame of all time slots, or adjustment of Q parameters according to empty slots and the amount of collision slots to open a new frame to identify the unknown label until the completion of all tag identification, and then end tag recognition processing. In Fig. 2, the FRAME1 frame successfully identified the Tag3 and Tag4 two tags, and left the Tag1, Tag2 and Tag5 three tags are not recognized. According to the literature [1], when the tags number to be identified is equal to the length of the frame, the recognition efficiency is the highest, so adjusted the frame length of the new frame FRAME2 to 3 by using the Adjust command, and the recognition of the next frame is carried out. The recognition process of FRAME2 frame is similar to that of FRAME1. In this case, the FRAME2 frame Slot1 time slot successfully identifies the Tag2, the Slot2 time slot successfully identifies the Tag1, and the Slot3 time slot successfully identifies the Tag5. In practical engineering the computation efficiency is considered, and the frame length is usually designed as an integer order of 2, which is convenient for computer processing. Adjustment algorithm of EPC_C1 G2 standard Adjust command adopted Q algorithm is shown in Fig. 3.

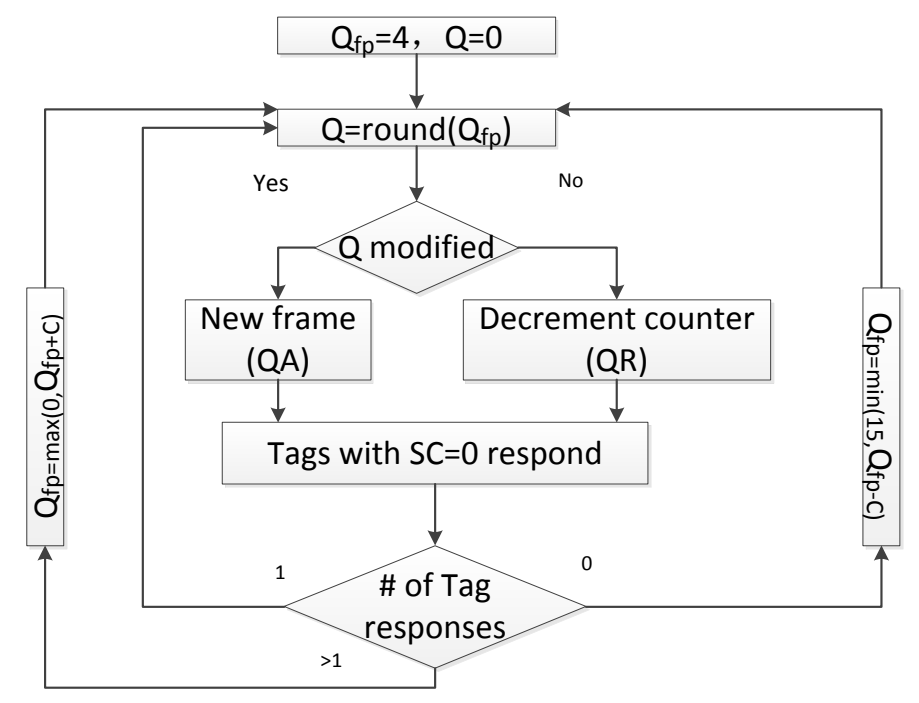

Figure 3. Q algorithm

If the $\mathrm{Q}$ value is large and tags number is small relatively, it is possible to generate more empty slots; instead, if the $Q$ value is small and tags number is large relatively, there may be more 
collisions. Therefore, the Q parameters need to be adjusted according to the practical application

\section{Conclusions}

The intelligent parking management system based on radio frequency identification technology is designed, including the system structure and the processing flow. The characteristics of the design is when multiple vehicles at the same time into the parking space, the use of group binding strategy is a good solution to the timing and billing, as well as parking management issues.

\section{Acknowledgements}

College students' innovative entrepreneurial training project, Project number: 127152016128. Teaching reform project of Xijing University, Project number: JGYB1641. Scientific research fund of Xijing University, Project number: XJ160140.

\section{References}

[1] Luca Barletta, Flaminio Borgonovo, Matteo Cesana. A formal proof of the optimal frame setting for Dynamic-Frame Aloha with known population size [J]. IEEE Transactions on information theory (S0018-9448), VOL. 60, NO. 11, NOVEMBER 2014:7221-7230.

[2] Lei Zhu, Tak-Shing Peter Yum. Optimal Framed Aloha Based Anti-Collision Algorithms for RFID Systems [J]. IEEE Trans on Communications (S0090-6778), 2010(58): 3583- 3592.

[3] Sanjay P. Ahuja, Varun Bhogal, Zornitza Genova Prodanoff, Kenneth Martin. On BFSA Collision Resolution in LF, HF, and UHF RFID Networks [J]. International Journal of Wireless Networks and Broadband Technologies (s2155-6261), 2015, 42.

[4] Zhang D, Li G, Pan Z, et al. A new anti-collision algorithm for RFID Tag [J]. International Journal of Communication Systems(s 1099-1131), 2013, 27 (11): 3312-3322.

[5] Yan X Q, Liu Y, Li B, et al. Numeric Evaluation on the System Efficiency of the EPC Gen-2 UHF RFID Tag Collision Resolution Protocol in Error Prone Air Interface[J]. International Journal of Distributed Sensor Networks(s 1550-1329), 2014 (50):1-9.

[6] Wu H, Zeng Y, Feng J, et al. Binary tree slotted ALOHA for passive RFID tag anticollision [J]. IEEE Transactionson Parallel and Distributed Systems (S1045-9219), 2013, 24(1): 19-31.

[7] Zhang Lijuan, Zhang Jin, Tang Xiaohu. Assigned Tree Slotted Aloha RFID Tag Anti-Collision Protocols [J].IEEE Transactions on Wireless Communications (S1536-1276), 2013, 12(11): 5493-5505.

[8] Di Giampaolo Emidio, Martinelli Francesco. A Passive UHF-RFID System for the Localization of an Indoor Autonomous Vehicle [J]. IEEE Transactions on Industrial Electronics (S0278- 0046), 2012, 59(10): 3961-3970.

[9] Zhenpeng L, Zhenyang G, Kaiyu S, et al. Anti-collision Algorithm for RFID Based on Continuous Collision Detection [J]. TELKOMNIKA Indonesian Journal of Electrical Engineering (S1693-6930), 2013, 11(12): 7476- 7483.

[10] Chin-Ling Chen, Mei-Li Chong. A radio frequency identification application for car theft prevention in parking lot management systems [J]. Int. J. Commun. Syst., 2014. 